\title{
COVID-19 Super Spreading Event Amongst Elderly Individuals — Jilin Province, China, January 2021
}

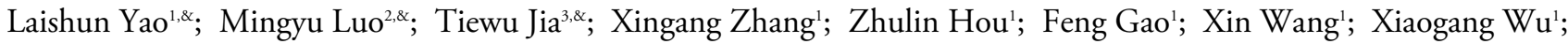 \\ Weihua Cheng'; Guoqian $\mathrm{Li}^{4}$; Jing Lu${ }^{4}$; Bing $\mathrm{Zhao}^{3}$; $\mathrm{Tao} \mathrm{Li}^{3}$; Enfu Chen²; Dapeng Yin ${ }^{3, *} ;$ Biao Huang ${ }^{1, * *}$
}

\begin{abstract}
Summary
What is already known on this topic?

Clusters of COVID-19 cases often happened in small settings (e.g., families, offices, school, or workplaces) that facilitate person-to-person virus transmission, especially from a common exposure.

What is added by this report?

On January 10 and 11, 2021, an individual gave three product promotional lectures in Tonghua City, Jilin Province, that ultimately led to a 74-case cluster of COVID-19. Our investigation determined the outbreak to be an import-related COVID-19 superspreading cluster event in which elderly, retired people were exposed to the infected individual during his promotional lectures, which were delivered in a confined space and lasted several hours.

What are the implications for public health practice?

Routine activities, such as attending a lecture in a classroom, can provide an environment conducive to COVID-19 superspreading events because respiratory viruses can spread easily and widely. We suggest local government to strengthen infection control management, reduce unnecessary indoor large gathering activities, and promote wearing of masks, especially during wintertime in the north of China. Health education for elderly people should promote use of effective personal protection and emphasize the importance of wearing masks.
\end{abstract}

On January 10, 2021, 4 people from Wangkui County, Suihua City, Heilongiiang Province returned to Jilin Province. They tested positive for coronavirus disease 2019 (COVID-19) and subsequently caused an outbreak in Tonghua City of Jilin Province (1). On January 11, Jilin Province initiated their COVID-19 prevention and control emergency plan. China CDC and Jilin CDC jointly conducted an epidemiological investigation and traced the outbreak. Based on the findings of the investigation, Dongchang District was locked down to help stop virus transmission starting from January 20.

\section{INVESTIGATION AND RESULTS}

Through January 31, 2021, there have been about 140 cases associated with the same case (called Mr. L in this report), showing Mr. L to be a super spreader.

Mr. L, a 44-year-old male, is a product promotion lecturer who travels often. From December 23, 2020 to January 3, 2021, Mr. L traveled by train and plane in Shandong, Shanxi, Henan, and Heilongjiang provinces; from January 3 to $6, \mathrm{Mr}$. L traveled by train inside Heilongjiang Province; and on January 7 , he traveled through Jilin Province by train to Changchun City. From January 9 to 12, Mr. L lived in Tonghua City and hosted product promotion training courses, dining in small restaurants, and having external contact. Mr. L took the K350 train on January 5, traveling in a seat adjacent to 2 of the aforementioned 4 confirmed cases from Wangkui County. He arrived in Tonghua on January 9 and conducted 3 training activities in the following 2 days. At noon on January 10, due to muscle pain and other symptoms, Mr. L went to a pharmacy (his body temperature was in the normal range when measured at the pharmacy) (Figure 1)

On January 10 and 11, Mr. L gave 3 lectures in Location A of Tonghua City, with each lecture lasting about 2.5 hours. The classroom of Location A was about 30 square meters and had doors and windows closed. Mr. L did not wear a mask during the 3 lectures. A total of 97 (40,31, and 26) participants took part in the 3 lectures. Most participants were retirees; $61 \%$ (59/97) were female; and the median age was 72 years (60 to 87 years). By January 31, 76\% (74/97) of the participants were tested positive. The attack rates in the 3 lectures were $90 \%(36 / 40)$, 90\% $(28 / 31)$, and $38 \%(10 / 26)$. In addition, Mr. L infected at least 7 other contacts, including 5 employees at Site A (Ms. D, in an hotel room next to Mr. L's room; and 
Ms. E, an employee at the restaurant that Mr. L visited twice). Infected close contacts of Mr. L spread the virus during dinners, other lectures, shopping, and playing mahjong, etc. There were more than 30 infections by the close contacts of Mr. L. In summary, until January 31, Mr. L directly infected 81 close contacts (firstgeneration cases) and indirectly infected at least 60 close contacts of the contacts (second-generation and third-generation cases) in Dongchang District of Tonghua (Figure 2).

On January 10, Jilin CDC sequenced the virus isolated from the first batch of pharyngeal swab samples from the original imported cases from Wangkui County, Suihua City, Heilongjiang Province. On January 12, the sequencing data were delivered to China CDC for whole genome sequencing. The 4

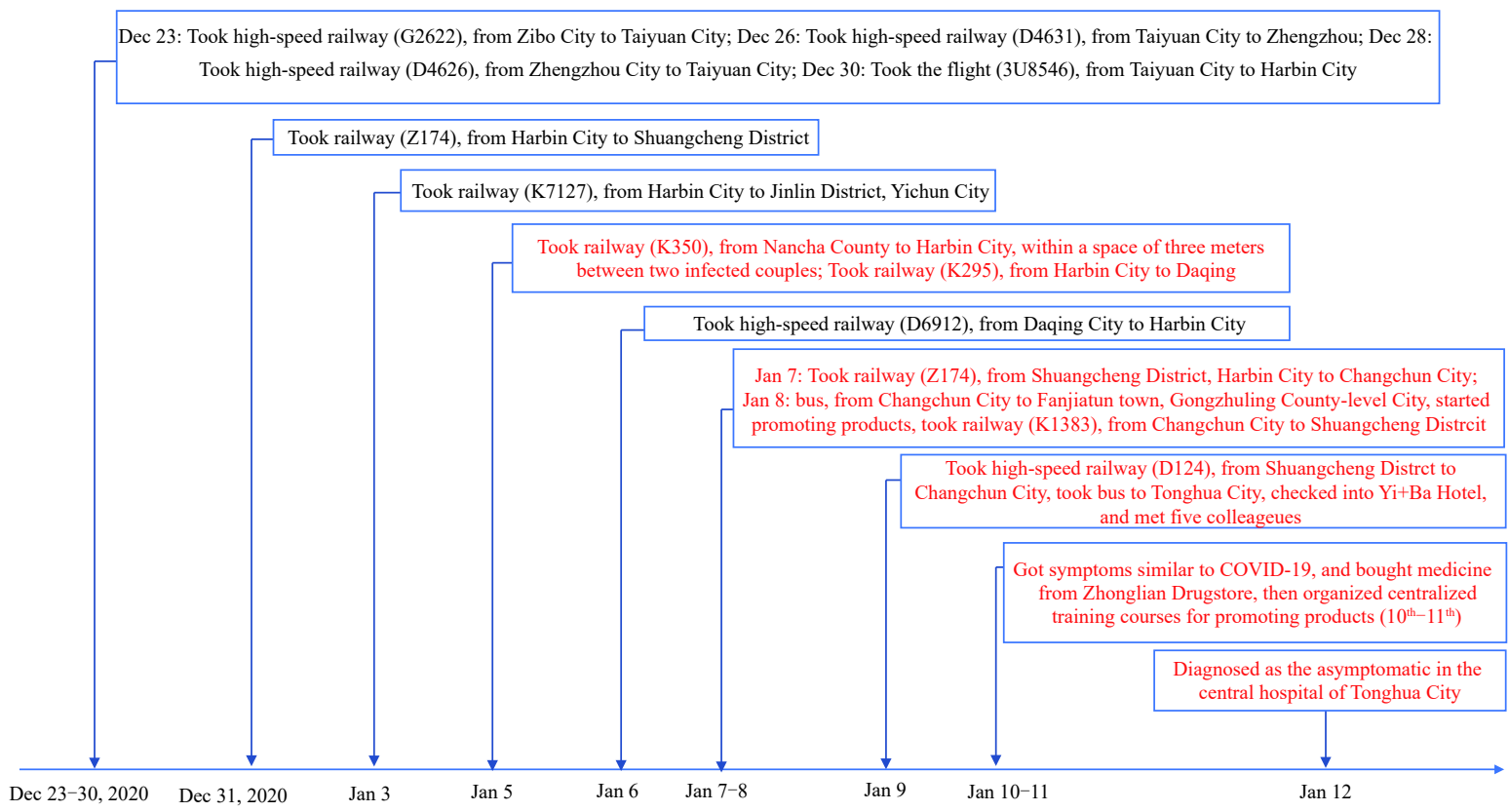

FIGURE 1. Movement of Mr. L from December 23, 2020 to January 12, 2021.

Legend

Color of spots:

: Index case

: First-generation cases

: Second-generation cases

: Third-generation cases

Line type:

- Participation in product promotion course

$\sim$ : Live together as family members

: Daily life contacts (such as neighbors)

.......: Others

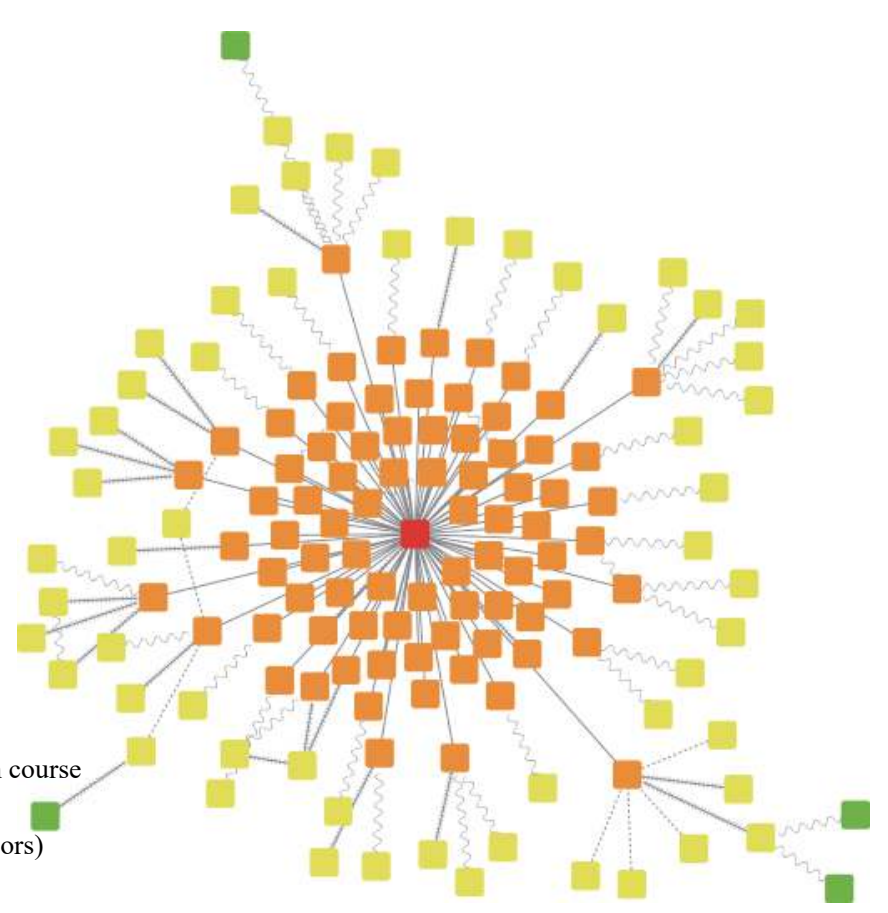

FIGURE 2. Three generations of infection from index case, Mr. L from January 12 to January 31, 2021. 
genome samples belonged to the L-genotype of the European I branch (b.1.1) and shared 12 nucleotide mutations with the first case of the December 2020 Dalian outbreak and the January 2021 Wangkui outbreak. On January 16, Jilin CDC provided a second batch of 11 samples (including Mr. L's sample) to China CDC for whole sequencing analysis. The sequences of the two batches were highly homologous and belonged to the same transmission chain.

\section{DISCUSSION}

The epidemic in Tonghua City was detected early but developed rapidly. The outbreak was directly related to Mr. L, and had a clear intergenerational transmission relationship.

Most of the individuals in the outbreak were retired elders from small and medium-sized cities. The joint epidemiological investigation found that these individuals lived a somewhat sedate lifestyle, had a similar socioeconomic level, and were eager to participate in external activities. They took free buses to other locations, greatly expanding their geographic range for activities. Because the course participants were elderly, the outbreak had a high proportion of severe and critical cases. By the end of January, 47 severe or critical cases were reported, accounting for $15.2 \%$ of the total cases, including 1 death of an $87-$ year-old person on January 25, 2021.

Three major factors contributed to this outbreak: First, Mr. L had muscle pain and visited a pharmacy on January 10 to obtain medication. This indicated that Mr. L was likely at an early stage of disease when holding lectures on January 10 and January 11. Early stage illness is believed to be most infectious (2). Second, most of the participants in his classes were elderly, with a median age of 72 years. The classes lasted long and were held in a small, confined space. Third, personal protection was not utilized, and social distancing were not maintained. The classroom was crowded, with about 30 people in a 30 square meters space. Mr. L and many participants did not wear masks during class. The outbreak was relatively confined, and no new virus strains were identified through genetic sequencing. This investigation showed, therefore, that there was no evidence of increased virulence or transmission.
In winter, the life of retired people in the north can be sedate and routine. Participating in educational events is an attractive activity. Thus, imported viruses can spread easily and widely. We suggest that local governments strengthen infection control management and reduce unnecessary indoor large gathering activities during wintertime, especially in the north of China. Health education for elderly people should be strengthened to promote use of effective personal protection.

Acknowledgements: Feng Tan, Xudong $\mathrm{Li}$, and Lance Rodewald from China CDC Weekly.

Feild teams on case and close contact tracing: Tonghua City CDC Team; Jilin Province CDC Team; Jilin City CDC Team; Jilin City, Changyi District CDC Team; Jilin City, Chuanying District CDC Team; Jilin City, Longtan District CDC Team; Jilin City, Fengman District CDC Team; Siping City CDC Team; Siping City, Lishu County CDC Team; Siping City, Shuangliao City CDC Team; Siping City, Yitong County CDC Team; Meihekou City CDC Team; Liaoyuan City CDC Team; Liaoyuan City, Dongfeng County CDC Team; Liaoyuan City, Dongliao County CDC Team; Baishan City CDC Team; Baishan City, Jiangyuan District CDC Team; Baishan City, Fusong County CDC Team; Baishan City, Jingyu County CDC Team; Baishan City, Linjiang County CDC Team; Changbai County CDC Team.

doi: $10.46234 /$ ccdcw 2021.050

\# Corresponding authors: Dapeng Yin, yindp@chinacdc.cn; Biao Huang, huangbiao2000@sohu.com.

\footnotetext{
${ }^{1}$ Jilin Center for Disease Control and Prevention, Changchun, Jilin, China; ${ }^{2}$ Zhejiang Center for Disease Control and Prevention, Hangzhou, Zhejiang, China; ${ }^{3}$ Chinese Center for Disease Control and Prevention, Beijing, China; ${ }^{4}$ Tonghua Center for Disease Control and Prevention, Tonghua, Jilin, China.

\& Joint first authors.
}

Submitted: February 06, 2021; Accepted: February 08, 2021

\section{REFERENCES}

1. Zhou L, Yao LS, Hao P, Li C, Zhao QL, Dong HL, et al. COVID-19 cases spread through the K350 train - Jilin and Heilongjiang Provinces, China, January 2021. China CDC Wkly 2021. http://dx.doi.org/10.46234/ ccdcw2021.026.

2. Kim MC, Cui CG, Shin KR, Bae JY, Kweon OJ, Lee MK, et al. Duration of culturable SARS-CoV-2 in hospitalized patients with Covid19. N Engl J Med 2021. http://dx.doi.org/10.1056/NEJMc2027040. 